\title{
DIRECT DETECTION OF PLANETS ORBITING LARGE ANGULAR DIAMETER STARS: SENSITIVITY OF AN INTERNALLY OCCULTING SPACE-BASED CORONAGRAPH
}

\author{
Justin R. Crepp ${ }^{1}$, Suvrath Mahadevan ${ }^{2}$, and Jian Ge \\ Department of Astronomy, University of Florida, 211 Bryant Space Science Center, Gainesville, FL 32611, USA; jcrepp@astro.caltech.edu \\ Received 2009 April 9; accepted 2009 June 30; published 2009 August 14
}

\begin{abstract}
High-contrast imaging observations of large angular diameter stars enable complementary science questions to be addressed compared to the baseline goals of proposed missions like the Terrestrial Planet Finder-Coronagraph, New World's Observer, and others. Such targets, however, present a practical problem in that finite stellar size results in unwanted starlight reaching the detector, which degrades contrast. In this paper, we quantify the sensitivity, in terms of contrast, of an internally occulting, space-based coronagraph as a function of stellar angular diameter, from unresolved dwarfs to the largest evolved stars. Our calculations show that an assortment of band-limited image masks can accommodate a diverse set of observations to help maximize mission scientific return. We discuss two applications based on the results: the spectro-photometric study of planets already discovered with the radial velocity technique to orbit evolved stars, which we elucidate with the example of Pollux $b$, and the direct detection of planets orbiting our closest neighbor, $\alpha$ Centauri, whose primary component is on the main sequence but subtends an appreciable angle on the sky. It is recommended that similar trade studies be performed with other promising internal, external, and hybrid occulter designs for comparison, as there is relevance to a host of interesting topics in planetary science and related fields.
\end{abstract}

Key words: instrumentation: adaptive optics - instrumentation: high angular resolution - methods: numerical planetary systems: formation - stars: evolution - stars: imaging

Online-only material: color figure

\section{INTRODUCTION}

High-contrast imaging is contingent upon the destructive interference of starlight. Losses in spatial coherence due to the finite size of a star, whose surface is comprised of many independently radiating elements, can limit the imaging sensitivity of a coronagraph. The effect is important when attempting to generate contrast levels that exceed several billions in reflected light.

A number of nearby stars have sizable angular diameters compared to the spatial resolution of a large optical telescope. Evolved stars, in particular, have intrinsically large radii and may subtend several instrument diffraction widths. For example, the largest star in the sky, R Doradus, is a red giant of diameter $D_{*}=57 \pm 0.5$ mas (Bedding et al. 1997). It illuminates an "area of coherence" (Born \& Wolf 1999), in quasi-monochromatic light centered on $\bar{\lambda}=0.55 \mu \mathrm{m}$, that is $0.6 \mathrm{~m}$ in diametersignificantly smaller than the primary mirror with which largestrategic or flagship status missions may operate (Beckwith 2008). For comparison, a $1 R_{\odot}$ star located at 10 pcs would have an angular diameter of $D_{*}=0.9$ mas and coherently illuminate an area of $40.3 \mathrm{~m}$ in diameter over the same bandpass.

An interesting regime lies between the spatially unresolved stars, where the performance of coronagraphs is nominally quoted, and large angular diameter giants, where performance is severely affected. This intermediate observational parameter space contains several dozen luminosity class I-IV stars and $\alpha$ Centauri A, whose proximity makes for an exceptional case.

\footnotetext{
1 Current Address: Caltech Optical Observatories/Department of Astronomy, California Institute of Technology, 1216 E. California Blvd. Pasadena, CA 91125, USA.

2 Current Address: Department of Astronomy \& Astrophysics, Pennsylvania State University, 525 Davey Lab, University Park, PA 16802, USA.
}

Such stars enable complementary science questions to be addressed compared to the goals of missions like the proposed Terrestrial Planet Finder-Coronagraph (TPF-C; Traub et al. 2007), New World's Observer (NWO; Cash 2006), Advanced Technology Large-Aperture Space Telescope (ATLAST; Stapelfeldt et al. 2009), and others, whose primary objective is to search for "Earth-like" planets orbiting F, G, K main-sequence stars.

For example, evolved stars can offer access to a different range in stellar mass and luminosity and thus opportunities for testing theories of planet formation and evolution in a variety of environments. Radial velocity surveys have recently begun to explore this territory. Their preliminary studies of the Jovian planet population around intermediate-mass stars have already revealed several apparent trends, including relationships between stellar mass and planet mass and period (Johnson et al. 2007, 2008). Several of these planets are directly detectable and warrant imaging confirmation and further characterization. One of the planets orbits a K0 III star, Pollux, which has an angular diameter of 8.0 mas.

The $\alpha$ Centauri system is located 1.4 pcs away from the Sun. Its primary components are the two highest priority targets on the TPF-C Top 100 list, ${ }^{3}$ but they have angular diameters of 8.6 and 6.0 mas. Special measures must be taken to observe such stars with the aforementioned coronagraphic missions, whose primary mirrors range from $D_{\text {tel }}=4-16 \mathrm{~m}$ in diameter, since a significant amount of starlight can leak around the coronagraphic mask and reach the detector before the stellar surface is fully resolved $\left(D_{*}<\lambda_{\min } / D_{\text {tel }}\right)$-an effect that we quantify in this paper.

To image a planet orbiting an extended source, an external occulter will need to cover (occult) more solid angle on the sky

\footnotetext{
3 http://planetquest.jpl.nasa.gov/TPF-C/.
} 
compared to that of a "point" source. This may be accomplished by decreasing the distance between the star shade and telescope (Lo et al. 2009). An internal occulter can switch masks in a focal plane wheel, but resolved patches of the stellar surface will fall onto different mask locations and create non-common-path errors that can only be partially corrected with a deformable mirror. Since there exist trade-offs between sensitivity, innerworking-angle (hereafter IWA), operating mode, duty-cycle efficiency, source brightness, signal-to-noise ratio $(\mathrm{S} / \mathrm{N})$, cadence, and other parameters, observational plans must account for the effect of stellar size to balance mission goals with mission lifetime. The fraction of time that a particular mission will dedicate to observations of large angular diameter stars is currently unclear. However, the $\alpha$ Centauri system places a firm non-zero lower limit.

While knowledge of instrument sensitivity as a function of stellar size is pertinent for prioritizing targets, especially if the observing strategy aims to be flexible and incorporate recent discoveries, the literature is currently devoid of such information for stars with large angular diameters. In this paper, we calculate the contrast that an internally occulting, spacebased coronagraph can generate as a function of stellar angular diameter for different operating modes. The particular design we model utilizes fourth and eighth-order band-limited image masks (Kuchner \& Traub 2002; Kuchner et al. 2005).

The effects of low-order optical aberrations on band-limited Lyot coronagraphs have been studied with analytic and numerical techniques (Shaklan \& Green 2005; Sivaramakrishnan \& Lloyd 2005; Crepp et al. 2007) and lab experiments (Crepp et al. 2006). Here, we present a full treatment of spatial incoherence that arises from resolving the stellar surface. Our end-to-end simulation tools explicitly model the dominant physical effects and incorporate the most recent wavefront correction algorithm: "electric-field conjugation" (Give'on et al. 2007).

Since large angular diameter stars represent an important family of high-contrast imaging targets, we present these results in anticipation of comparing them to other promising coronagraphic designs, like PIAA (Guyon 2003), distant and not-so-distant external occulters (Cash 2006; Spergel et al. 2009), interferometric coronagraphs (Shao 2009), shaped pupils (Kasdin et al. 2003), apodized pupils (Soummer 2005), vortex masks (Mawet et al. 2007), and others; each can, in theory, compensate for stellar size. Guyon et al. (2006) have compared these designs on an equal footing using a "useful throughput" metric, but only for sources with an extent of $0.2 \lambda / D_{\text {tel }}$. Also, information on wavefront quality and correction capabilities must be supplied to convert useful throughput into contrast. In the following, we calculate the intensity of residual starlight within the dark-hole search area over the entire range of stellar angular diameters, from unresolved dwarfs to the largest evolved stars. These numerical simulations can be used to facilitate feasibility studies for current and future science cases. Based on the results, we briefly discuss two applications within the context of extrasolar planet imaging.

\section{NUMERICAL SIMULATIONS}

We model a TPF-C-like instrument with code written in Matlab assuming an internally occulting Lyot-style design. Diffraction is managed with band-limited image masks (Kuchner \& Traub 2002; Kuchner et al. 2005). The telescope is circular and unobscured. Simulations are broadband and incorporate primary mirror phase and amplitude errors, image mask phase errors, a single, noiseless $64 \times 64$ actuator deformable mirror (DM), and the finite size of the star.

Stars are modeled with a uniform disk of mutually incoherent point sources. The effects of limb-darkening are also explored in a single case. Light from each source is sent through the optical train with a tip/tilt error that corresponds to its location on the stellar surface. The number of sources across the disk well exceeds the Nyquist frequency in $\lambda_{\min } / D_{\text {tel }}$ units. The intensities from each add together at the detector to form the final image. Ten wavelength channels sample each of three different 100 $\mathrm{nm}$ wide bandpasses. All optics are located in a pupil or image plane and Fourier transforms are used to propagate the electric field. We refer the reader to Shaklan \& Green (2006) for a discussion of Fresnel propagation effects, such as phase-induced amplitude aberrations and amplitude-induced phase aberrations, which can limit a coronagraph's broadband performance. They derive optical quality requirements for a contrast level of $10^{-12}$ per spatial frequency at visible wavelengths over the same bandpass - an order of magnitude fainter than the scattered light levels dealt with in this study.

Primary mirror phase errors follow a broken power-law power-spectral-density (PSD) given by

$$
\operatorname{PSD}(k)=\frac{A_{0}}{1+\left(k / k_{0}\right)^{n}},
$$

where $A_{0}=9.6 \times 10^{-19} \mathrm{~m}^{4}, k$ is the spatial frequency, $k_{0}=4$ cycles $\mathrm{m}^{-1}$, and $n=3$. This is the PSD typical of an $8 \mathrm{~m}$ primary mirror (Shaklan \& Green 2006; Bordé \& Traub 2006). The mirror surface figure is scaled to have an root-mean-square (rms) value of $1 \mathrm{~nm}$ ( $2 \mathrm{~nm}$ in wavefront phase). Amplitude errors are modeled as white noise with an rms of 0.005 and maximum value of unity. We do not model the effects of polarizationinduced low-order aberrations (Elias et al. 2004; Breckinridge \& Oppenheimer 2004), which can be suppressed to contrast levels below $10^{-10}$ for point sources with an eighth-order mask (Shaklan \& Green 2005), nor scattering from other optics in the path other than the glass on the image mask.

Mask defects, such as phase shifts that result from the manufacturing process, can limit the achievable contrast in polychromatic light for point sources (Lay et al. 2005; Balasubramanian 2008; Moody et al. 2008). Resolved sources add an additional complication in that different sections of the star's surface fall onto different mask locations and create non-common-path errors that cannot each be compensated for simultaneously; correcting one error may amplify another. We incorporate these effects into our simulations by modeling mask imperfections as phase errors that are correlated on a scale of $0.25 \lambda_{\text {min }} / D_{\text {tel }}$.

We use both fourth- and eighth-order linear band-limited masks to make a comparison study since they have a different resistance to stellar size. Their amplitude transmissions follow $\operatorname{sinc}(. .)^{2}$ and $\operatorname{sinc}(.)+.\operatorname{sinc}^{2}(.$.$) profiles, respectively (see$ Kuchner et al. (2005) for details). The default IWA is $4 \lambda_{\max } / D_{\text {tel }}$.

The DM is placed at a pupil and its surface is shaped by a square grid of actuators that map perfectly onto the primary mirror. The influence of each actuator is modeled with a Gaussian function that drops to $6 \%$ of its peak value at the location of adjacent actuators. The $64 \times 64$ system can correct for wavefront errors with spatial frequencies as high as 32 cycles per aperture. This creates the familiar "dark-hole" region in the image plane that defines the search area (Trauger et al. 2004; Trauger \& Traub 2007). We sacrifice correction of the highest spatial frequencies, 30-32 cycles per aperture, 
to improve correction of lower-order modes, further reducing the intensity of speckles close to the optical axis. A smaller search area can yield even deeper contrast, but here we are also interested in distant planets.

We calculate the optimal DM shape using the "electric-field conjugation" algorithm developed by Give'on et al. (2007). The technique is quite general and produces deeper darkholes compared to previous techniques, like speckle-nulling, by a factor of order unity. Successful implementation relies upon accurate modeling of the coronagraph, electric field reconstruction at the science camera, and a form of phase diversity to solve for the actuator heights. The procedure is efficient once a rather computationally expensive metric, the "G-matrix," is established for the optical system. It needs calculating only once, unless changes to the coronagraph are made. In this paper, we use several different coronagraphs, of different mask order, bandpass, IWA, and size of mask phase errors, so it was necessary to utilize multiple processors in parallel (but with no message passing interface).

The optimal DM shape is found using only the central (onaxis) portion of the star. The surface is then fixed-a DM cannot compensate for stellar size-and contrast is measured as a function of angular diameter. In practice, wavefront sensing may be performed on a main-sequence star with small angular diameter; then the telescope can slew to the target of interest.

Results use information from a single image. The instantaneous contrast, $C(x, y)$, is found using the formula in Green \& Shaklan (2003) and Crepp et al. (2007), which we show here:

$$
C(x, y)=\frac{I(x, y)}{I(0,0)|M(x, y)|^{2}},
$$

where $I(x, y)$ is the intensity at the coordinates $(x, y)$ in the final image, $I(0,0)$ is the peak stellar intensity that would be measured without the image mask in the optical train, and $|M(x, y)|^{2}$ is the mask intensity transmission. Both $I(x, y)$ and $I(0,0)$ are measured with the Lyot stop in place. Linear masks have no dependence on $y$. Below, we evaluate contrast by taking the median value of $C(x, y)$ in a $\lambda_{\min } / D_{\text {tel }}$ wide box whose inneredge is located at the IWA in the most sensitive half of the dark hole.

\section{CONTRAST VERSUS ANGULAR DIAMETER}

We compare fourth and eighth-order masks in systems optimized for two different bandpasses at visible wavelengths, $\lambda=0.5-0.6 \mu \mathrm{m}$ and $\lambda=0.7-0.8 \mu \mathrm{m}$, and one in the nearinfrared, $\lambda=2.2-2.3 \mu \mathrm{m}$. The latter bandpass is included since planets may exhibit interesting spectral features in the nearinfrared (Kaltenegger et al. 2007; Beckwith 2008, and references therein) and there are several practical benefits regarding dark-hole depth and width as the wavelength increases. We also include a mask with a larger IWA and place limits on the size of mask phase errors as a function of stellar diameter.

Results from our simulations are shown in Figure 1, where we plot contrast versus angular diameter for stars wider than 0.2 mas. The smallest, median, and largest targets from the TPF-C Top 100 list and the largest star in the sky, R Doradus, are shown for comparison. The upper horizontal axis of each plot also indicates the characteristic diameter of a variety of stars placed at 15 pcs. A filled star denotes the approximate location of Pollux $b$ in reflected light (Section 4.1).

The top panel of Figure 1 compares fourth and eighth-order masks in different bandpasses. Each has an IWA of $4 \lambda_{\max } / D_{\text {tel }}$.
In the small star regime, where $D_{*} \ll \lambda_{\min } / D_{\text {tel }}$, contrast is limited by DM fitting errors, and the sensitivity to stellar size is constant over most of the TPF-C target list. Further increases to $D_{*}$, however, show that all masks leak starlight before the stellar surfaces are fully resolved: $\lambda_{\min } / D_{\text {tel }}=14.2,19.3$, and 58.0 mas for each bandpass respectively. In this regime, where $D_{*} \sim \lambda_{\min } / D_{\text {tel }}$, the eighth-order mask has a significantly higher tolerance to stellar size than the fourth-order mask. This result makes intuitive sense since the eighth-order mask is also less susceptible to tip/tilt errors (Kuchner et al. 2005; Shaklan \& Green 2005; Crepp et al. 2006, 2007). The difference between the effects, however, is twofold: (1) a star delivers a collection of tip/tilt errors to the coronagraph, each with a contribution to the diffracted light noise floor that depends on distance from the optical axis, and (2) the speckle halo from each source on the stellar surface will couple differently to mask errors. Our simulations (Section 2) are able to capture such details.

We find that an eighth-order mask is required to optimize sensitivity to the evolved star Pollux, which is known to host a long period planet, and the largest TPF-C star, $\alpha$ Cen A, which has a high-target priority (see Section 4). In the extreme case of R Doradus, instantaneous contrast levels of order $10^{-7}$ and $10^{-10}$ at $4 \lambda_{\max } / D_{\text {tel }}$ are possible with an eighth-order mask for visible and near-infrared applications, respectively.

For small stars, the contrast improves at longer wavelengths according to the scaling relation $C \propto\left(\lambda_{0} / \lambda\right)^{2}$, where $\lambda_{0}$ and $\lambda$ are the central wavelength of the original and current bandpass, respectively. The sensitivity in the near-infrared is an order of magnitude deeper than in the visible, at the expense of a factor of 3-4 in spatial resolution. This trade-off is well justified for planets with large orbital separations (Section 4.1). The relative improvement grows to several orders of magnitude with further increases in stellar angular diameter. Ground-based instruments will be able to probe the $C \geqslant 10^{-7}$ in the near-infrared over all stellar sizes with extreme adaptive optics (Macintosh et al. 2006; Dekany et al. 2007; Beuzit et al. 2006).

Limb darkening becomes important when the stellar surface is resolved. In this regime, the contrast improves by a factor of order unity compared to that of a uniform disk, since the outermost portions of the star-those that contribute most to the noise floor-are less intense (Wade \& Rucinski 1985). The second $\lambda=0.7-0.8 \mu \mathrm{m}$, eighth-order mask contrast curve assumes a radial intensity profile that is gray (the same for all wavelengths in the band) and decreases linearly away from the star center point. The relative intensity at the edge of the star was set to 0.6 , the value determined by a recent interferometric study of the red giant star $\epsilon$ Oph (Mazumdar et al. 2009). Limbdarkening coefficients are smaller in the near-infrared (Claret 2000) and thus the effects less pronounced.

The bottom panel of Figure 1 compares eighth-order masks with rms surface errors of $0.5,2.3$, and $5.0 \mathrm{~nm}$. An eighthorder mask with an IWA of $7.27 \lambda_{\max } / D_{\text {tel }}=150$ mas is also shown. Each was optimized for the $0.7-0.8 \mu \mathrm{m}$ bandpass. The $\mathrm{rms}=0.5 \mathrm{~nm}, \mathrm{IWA}=4 \lambda_{\max } / D_{\text {tel }}$ curve is the same from the upper panel. Increasing the size of mask errors scatters more light into the dark hole. For unresolved stars, the contrast scales according to $C \propto\left(\sigma / \sigma_{0}\right)^{2}$, where $\sigma_{0}$ and $\sigma$ are the total rms error from the mask and primary mirror added in quadrature for two different cases. Presumably, other uncorrelated errors, such as polarization effects, may also be added in quadrature to estimate how the scattered light noise floor may change. In this sense, Figure 1 serves as a calibration point for calculating the contrast of systems with different error budgets. The coronagraphic mask 

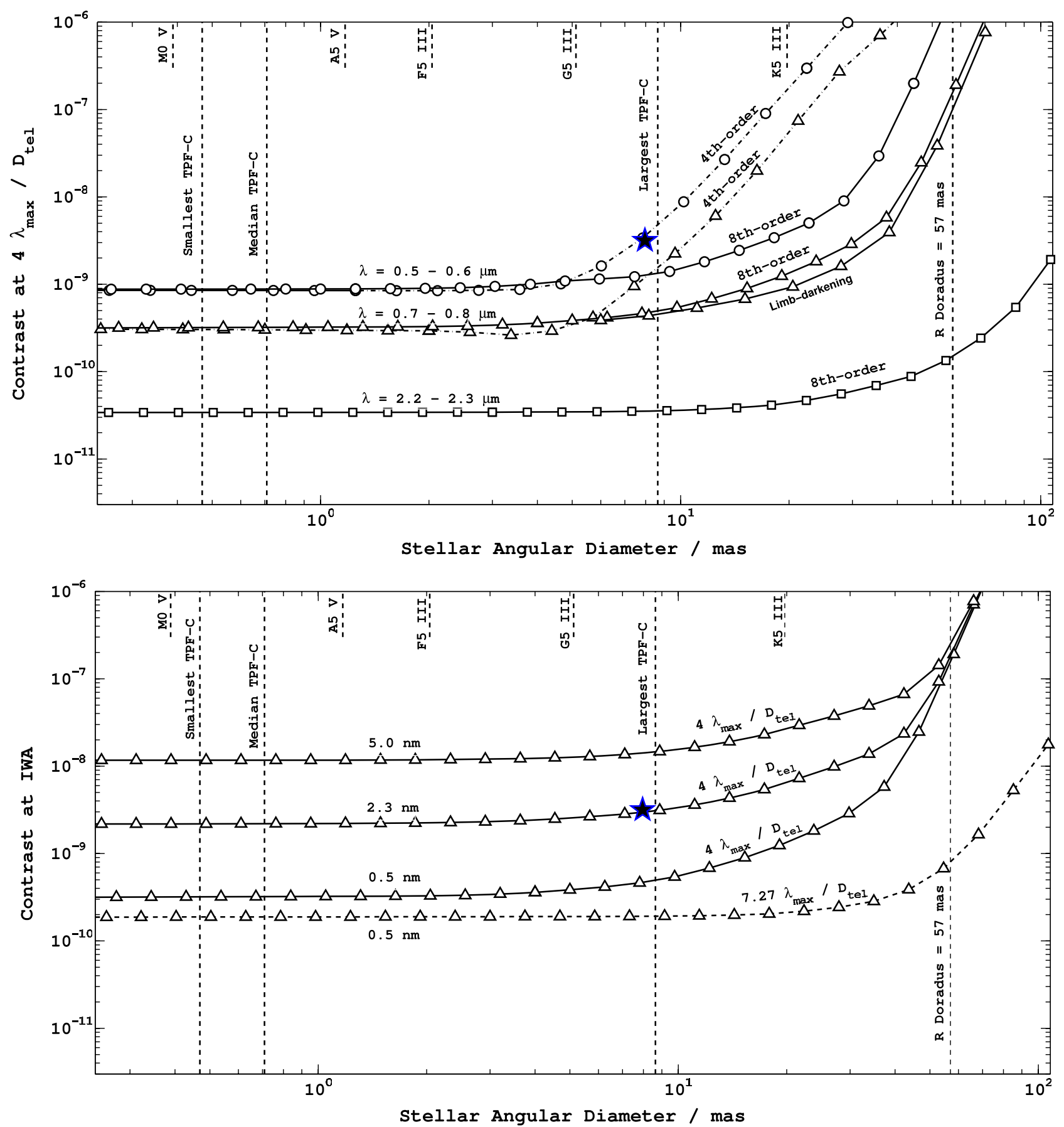

Figure 1. Coronagraph sensitivity to stellar angular diameter for an $8 \mathrm{~m}$ telescope. The characteristic size of various stars placed at 15 pcs are shown across the top axis in each graph for reference. We also include the diameter of R Doradus, the largest star in the sky, and entries from the TPF-C Top 100 List. A filled star denotes the reflected light contrast of the Jovian planet orbiting Pollux. (Top) Fourth and eighth-order mask performance in three different bandpasses. Different data points (circles, triangles, squares) denote different bandpasses. The size of mask phase errors is $0.5 \mathrm{~nm}$. The largest TPF-C star also has the highest priority (see Section 4.2 on $\alpha$ Centauri A). Limb-darkening is considered for the $\lambda=0.7-0.8 \mu \mathrm{m}$, eighth-order mask case. (Bottom) Performance of an eighth-order mask with different size mask phase errors and a design with an IWA of $7.27 \lambda_{\max } / D_{\text {tel }}$, each optimized for the $\lambda=0.7-0.8 \mu$ m bandpass.

(A color version of this figure is available in the online journal.)

with IWA $=7.27 \lambda_{\max } / D_{\text {tel }}$ passes more planet light and is also less susceptible to stellar size (see Section 4.1).

In practice, an internal occulter will need to implement post-processing techniques after creating a dark hole. However, the improvement in sensitivity of a coronagraph that utilizes differential imaging is nearly constant as a function of stellar size. We have verified this assertion with simulations of point-spread-function (PSF) roll-subtraction for stars as large as R Doradus and telescope diameters as large as $8 \mathrm{~m}$. Figure 1 can thus also be used to estimate the planet-imaging sensitivity of an instrument with speckle-discrimination capabilities.

The effect of stellar size will become increasingly problematic with time as telescope diameters and interferometric baselines continue to grow. To first order, we can scale the curves in Figure 1 according to telescope size, if we assume a constant 

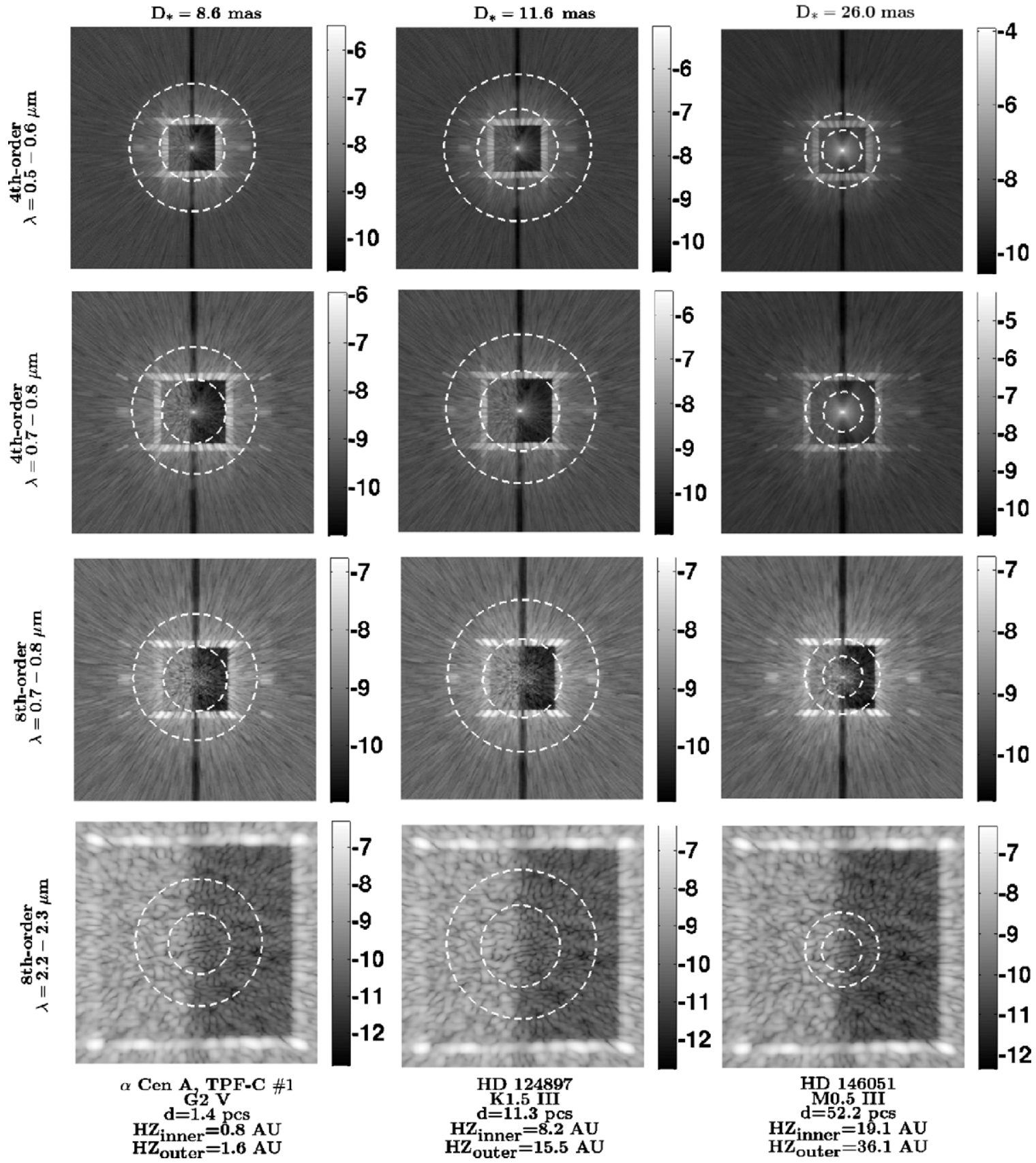

Figure 2. High-contrast images of three stars with large angular diameters. Each are more massive than the Sun. The gray scale shows the value of log $(I(x, y) / I(0,0))$. The dashed white lines indicate the location of the habitable zone for reference. Amplitude errors break the spatial symmetry of speckles allowing optimal suppression of only half of the dark-field with a single deformable mirror. The fourth-order mask leaks a significant amount of starlight. Observations in the near-infrared provide a wider dark-hole and deeper contrast, thus permitting the reflected light detection of more distant planets.

actuator density and neglect differences in the manufacturing processes that modify the PSD and thus the speckle pattern. For instance, a larger telescope with a correspondingly larger number of actuators would better resolve the stellar surface, leak more light, and result in a horizontal curve shift (to the left) by a factor of $8 / D_{\text {tel }}(\mathrm{m})$. Telescope designs with primary mirror diameters in excess of $8 \mathrm{~m}$, which may eventually be required to search a statistically significant sample of stars for terrestrial planets in reflected light (Beckwith 2008), are thus presented with a dilemma.

Figure 2 displays a series of high-contrast images for three nearby stars of increasing angular diameter. The current inner and outer-edge of the habitable zone for each is overlaid (dashed white lines) for reference. Only half of the dark-hole is accessible since we include primary mirror amplitude errors and a single deformable mirror. The gray scale indicates the value of $\log (I(x, y) / I(0,0))$. The images qualitatively verify the results from Figure 1. Stellar leakage is a serious problem for the fourth-order mask in both visible bandpasses, whereas the eighth-order mask is able to suppress starlight from extended sources to more acceptable levels. Sacrificing spatial resolution by conducting observations in the near-infrared improves the coronagraph's resistance to stellar size and dark-hole depth and width. Equipping a space-based coronagraph with a nearinfrared camera would increase the $\mathrm{S} / \mathrm{N}$ of spectra, enable the detection of planets at larger separations, and complement visible light observations. We next discuss two applications based on these results. 


\section{POTENTIAL APPLICATIONS}

\subsection{Evolved Stars}

Stars supply the material out of which planets originate. One might therefore expect some diversity in the statistical properties of planets, given that the range of masses over the zero-agemain-sequence typically spans 3 orders of magnitude. Studies of the planet population as a function of stellar mass may provide insight into the processes that govern their formation. A similar argument can be made with respect to the time evolution of planets. Planetary atmospheres are subject to the radiation they receive from their host star and stellar luminosities can vary by as many as 7 orders of magnitude over a lifetime, when the red giant to early compact object stages of evolution are included (Iben 1967; Iben \& Laughlin 1989). Combining this information suggests that evolved stars may become important targets for planet searches, if they are not already. Direct imaging can offer an efficient approach for probing this parameter space, as has been recently demonstrated by the simultaneous discovery of three planets orbiting HR 8799 (Marois et al. 2008).

A low-risk approach for large-aperture missions to target evolved stars is to follow-up planets that have already been detected with other techniques, like radial velocity and astrometry. An example of such a system with a known exoplanet is the bright ( $V=1.15$ ) K0 III giant $\beta$ Gem (Pollux). Radial velocity observations spanning nearly 25 years are consistent with the presence of a $M \sin i=2.6 M_{\text {Jup }}$ planet with a semimajor axis of 1.64 AU (Hatzes et al. 2006). Pollux is located at a distance of $10.3 \mathrm{pcs}$ and has a mass of $1.7 \pm 0.4 M_{\odot}$ (Allende-Prieto $\&$ Lambert 1999) and angular diameter of $7.96 \pm 0.09$ mas (Nordgren et al. 2001). The approximate location of Pollux b is labeled with a star in Figure 1. According to the curves, an eighth-order mask is required to unambiguously detect its presence at $\lambda=0.5-0.6 \mu \mathrm{m}$, assuming half illumination, a planet radius of $1 R_{\mathrm{Jup}}$, and albedo of 0.3 . The orbits of such planets will already be determined, and the combination of photometry and spectra with indirect techniques will place tight constraints on their mass.

We also note that terrestrial planets can be imaged in the extended habitable zones of evolved stars. A recent study by Lopez et al. (2005) has shown that there are eras of post-mainsequence evolution where hospitable conditions may persist for $10^{8}-10^{9}$ years. Ten percent of the stars within 30 pcs of the Sun that they recommend for a targeted search have angular diameters between $3 \lesssim D_{*} \lesssim 57$ mas. This same set of stars from their sample also have the brightest visual magnitudes, so may offer high observational efficiency.

Although distant terrestrial planets will generally be fainter than the Earth-Sun system in reflected light (contrast $\approx 2 \times$ $10^{-10}$ ), depending on their albedo and radius (see Seager et al. (2007) for a discussion of super-Earths), a coronagraph can accommodate by sacrificing spatial resolution. Observations at longer wavelengths decrease the intensity of speckles by a factor of $\sim\left(\lambda / \lambda_{0}\right)^{2}$ in the search area (Section 3). Another option is to increase the IWA (Figure 1 -bottom panel). With a bandlimited mask design, this results in higher Lyot stop throughput, which increases the amount of companion light, decreases integration time, and makes the PSF more spatially succinct. ${ }^{4}$ Notice that both approaches, and combinations thereof, improve the coronagraph's resistance to stellar size.

\footnotetext{
4 Highly concentrated PSF's facilitate discrimination of companion light amongst diffuse zodiacal and exozodiacal dust emission.
}

Table 1

Physical Parameters of the $\sim 5$ Gyr Old $\alpha$ Cen System

\begin{tabular}{lccc}
\hline \hline \multicolumn{1}{c}{$\alpha$ Centauri } & $\mathrm{A}$ & $\mathrm{B}$ & $\mathrm{C}$ \\
\hline Spec. Type & $\mathrm{G} 2 \mathrm{~V}$ & $\mathrm{~K} 1 \mathrm{~V}$ & $\mathrm{M} 5.5 \mathrm{~V}$ \\
V mag & -0.01 & 1.35 & 11.1 \\
Mass $\left(M_{\odot}\right)$ & 1.11 & 0.93 & 0.12 \\
Radius $\left(R_{\odot}\right)$ & 1.24 & 0.87 & 0.15 \\
Luminosity $\left(L_{\odot}\right)$ & 1.60 & 0.45 & 0.0002 \\
{$[\mathrm{Fe} / \mathrm{H}]$} & 0.22 & 0.26 & -1.00 \\
$\mathrm{a}(\mathrm{AU})$ & 23.4 & 23.4 & $\approx 12,000$ \\
$\mathrm{e}$ & 0.52 & 0.52 & $?$ \\
$\mathrm{i}$ & 79.2 & 79.2 & $?$ \\
Diameter (mas) & 8.6 & 6.0 & 1.0 \\
TPF-C Rank & $\# 1$ & $\# 2$ & $\ldots$ \\
\hline
\end{tabular}

\section{2. $\alpha$ Centauri}

The primary components of the $\alpha$ Centauri (Cen) triple star system are on the main sequence but have angular diameters of 8.6 and 6.0 mas, respectively (Table 1). They are the two highest priority targets on the TPF-C Top 100 List, where priority is defined by the first-visit completeness per unit integration time. The third component, Proxima Centauri, is a distant $\mathrm{M}$ dwarf that appears to be weakly bound (Wertheimer \& Laughlin 2006).

Numerical simulations show that terrestrial planets located in the habitable zone of $\alpha$ Cen $\mathrm{A}, \mathrm{B}$ may remain stable for timescales comparable to the system age, provided the inclination angle between the stellar and planetary orbital planes is sufficiently small (Wiegert \& Holman 1997; Quintana et al. 2007). The topic of whether terrestrial planets can form in their habitable zone in the first place though is still debated (Barbieri et al. 2002; Thébault et al. 2008, 2009). Imaging observations of intermediately spaced binaries can provide important data to further the development of planet formation models.

A vertical dashed line denotes the location of $\alpha$ Cen $\mathrm{A}$ in Figure 1 as the largest TPF-C star. We find that an eighthorder mask generates deeper contrast at the IWA than a fourthorder mask by factors of 3.1 and 3.6 at $\lambda=0.5-0.6 \mu \mathrm{m}$ and $\lambda=0.7-0.8 \mu \mathrm{m}$, respectively. The first column of Figure 2 shows simulated images of $\alpha$ Cen A in various observing modes and bandpasses. Given the relationship between wavelength and dark-hole depth and width (Section 3), planets in the habitable zone of $\alpha$ Cen A may be easier to detect in the near-infrared.

\section{CONCLUDING REMARKS}

Our primary objective with this work has been to quantify the high-contrast imaging sensitivity of an internally occulting, space-based coronagraph as a function of stellar angular diameter. Figures 1 and 2, illustrate the fundamental trade-offs associated with fourth and eighth-order band-limited masks in various bandpasses and with different size mask phase errors and IWA's for all stars in the sky. We have also included a case that incorporates the effects of limb darkening. These results can be used to develop observing strategies and new science cases for a TPF-C-like instrument, and may be compared to other promising internal, external, and hybrid occulter designs. We also note that NIRCAM in James Webb Space Telescope (JWST) will utilize band-limited image masks (Green et al. 2005).

A significant amount of starlight leaks through the coronagraph before the stellar surface is fully resolved. Consequently, 
many important targets will require non-default observational operating modes to accommodate for stellar size. An eighthorder mask can provide this leverage by generating as much as 2 orders of magnitude deeper contrast than a fourth-order mask for large angular diameter stars.

We also find that dark-hole depth depends sensitively on the optical quality of the mask, bandpass, and IWA. Only $5 \mathrm{~nm}$ of rms surface roughness (peak-to-valley $\approx 25 \mathrm{~nm}$ ) can degrade contrast to $\sim 10^{-8}$, prior to post-processing techniques. Nearinfrared observations can help to offset the effect by reducing the amount of scatter from optical components. They also offer a wider dark hole and thus may be ideal for detecting the reflected light of distant planets. There are several dozen targets where this newly afforded search space corresponds to the habitable zone. Most are evolved stars. Sacrifices in IWA can offer similar performance at the expense of search area.

There are two applications that follow immediately from these results. $^{5}$

1. Jovian planets have been detected orbiting evolved stars with the radial velocity technique. Their physical properties may be further constrained with spectro-photometric follow-up. The planet orbiting Pollux is an example and requires an eighth-order mask for unambiguous detection. Coronagraphs with intrinsically small IWA's may not be able to detect Pollux $b$ in reflected light. Presumably, more such planets will be detected in the future as the time baselines of radial velocity and astrometric searches increase.

2. Given its proximity to the Sun, $\alpha$ Centauri will be a highpriority target for any space-based high-contrast imaging mission (e.g., Kaltenegger et al. 2008). Both of its mainsequence stars have a large angular diameter. An eighthorder mask can generate a factor of $\sim 3$ deeper contrast at the IWA than a fourth-order mask for $\alpha$ Centauri A. Near-infrared observations are likely required to search its habitable zone with deformable mirrors that utilize $64 \times$ 64 actuators or less.

No other TPF-C stars require an eighth-order mask, unless a telescope with primary mirror in excess of $8 \mathrm{~m}$ in diameter is launched (e.g., ATLAST). Figure 1 can be used as calibration for scaling arguments to estimate the expected performance of different aperture sizes. Small-strategic or discovery-class missions, like ACCESS (Trauger et al. 2009), PECO (Guyon et al. 2009), DAVINCI (Shao 2009), EPIC (Clampin 2009), or THEIA (Spergel et al. 2009), should be able to observe large angular diameter stars without substantive losses in sensitivity, although will still require eighth-order masks for a number of targets. The former application may be a valuable investment of observing time given that such missions will be limited primarily to the characterization of Jovian planets, based on arguments of telescope collecting area and number of stars surveyed (Beckwith 2008). Moreover, it appears that massive stars tend to have more massive planets at larger separations (Johnson et al. 2008).

Given the scientific motivation for observing large angular diameter stars, it is reasonable that space-based high-contrast imaging missions consider a more comprehensive set of targets than the canonical list of F, G, K main-sequence stars. Otherwise, a limited scope or severe predisposition to certain kinds of stars neglects large classes of interesting systems, including those

\footnotetext{
5 Other applications include stellar multiplicity studies and the detection and characterization of brown dwarfs and debris disks.
}

that may tell us where to look next. Indeed, history has shown that this line of reasoning also tends to maximize the chances for serendipitous discovery (Angel 2001).

We thank Stuart Shaklan and Amir Give'on for helpful comments regarding an earlier draft of this manuscript and implementation of the electric field conjugation algorithm. We are also grateful for suggestions made by the referee that lead to improved presentation of our results. This work was supported by the UCF-UF SRI program and NASA grant NNG06GC49G.

\section{REFERENCES}

Allende-Prieto, C., \& Lambert, D. L. 1999, A\&A, 352, 555

Angel, R. 2001, Nature, 409, 427

Balasubramanian, K. 2008, Appl. Opt., 47, 116

Barbieri, M., Marzari, F., \& Scholl, H. 2002, A\&A, 396, 219

Beckwith, S. V. W. 2008, ApJ, 684, 1404

Bedding, T. R., Zijlstra, A. A., von der Luhe, O., Robertson, J. G., Marson, R. G., Barton, J. R., \& Carter, B. S. 1997, MNRAS, 286, 957

Beuzit, J.-L., et al. 2006, The Messenger, 125, 29

Bordé, P. J., \& Traub, W. A. 2006, ApJ, 638, 488

Born, M., \& Wolf, E. 1999, Principles of Optics (7 ed.; Cambridge: Cambridge Univ. Press)

Breckinridge, J. B., \& Oppenheimer, B. R. 2004, ApJ, 600, 1091

Cash, W. 2006, Nature, 442, 51

Clampin, M. 2009, BAAS, 41, 506

Claret, A. 2000, A\&A, 363, 1081

Crepp, J. R., Ge, J., Vanden Heuvel, A. D., Miller, S. P., \& Kuchner, M. J. 2006, ApJ, 646, 1252

Crepp, J. R., Vanden Heuvel, A. D., \& Ge, J. 2007, ApJ, 661, 1323

Dekany, R., et al. 2007, in Proc. Advanced Maui Optical and Space Surveillance Technologies Conf., ed. S. Ryan, E64

Elias, II, N. M., Bates, R., \& Turner-Valle, J. 2004, Proc. SPIE, 5555, 248

Give'on, A., Belikov, R., Shaklan, S., \& Kasdin, J. 2007, Opt. Express, 15 12338

Green, J. J., Beichman, C., Basinger, S. A., Horner, S., Meyer, M., Redding,

D. C., Rieke, M., \& Trauger, J. T. 2005, Proc. SPIE, 5905, 185

Green, J. J., \& Shaklan, S. B. 2003, Proc. SPIE, 5170, 25

Guyon, O. 2003, A\&A, 404, 379

Guyon, O., Pluzhnik, E. A., Kuchner, M. J., Collins, B., \& Ridgway, S. T. 2006, ApJS, 167, 81

Guyon, O., et al. 2009, BAAS, 41, 345

Hatzes, A. P., et al. 2006, A\&A, 457, 335

Iben, I. J. 1967, ARA\&A, 5, 571

Iben, I. J., \& Laughlin, G. 1989, ApJ, 341, 312

Johnson, J. A., Marcy, G. W., Fischer, D. A., Wright, J. T., Reffert, S., Kregenow, J. M., Williams, P. K. G., \& Peek, K. M. G. 2008, ApJ, 675, 784

Johnson, J. A., et al. 2007, ApJ, 665, 785

Kaltenegger, L., Eiroa, C., \& Fridlund, C. V. M. 2008, arXiv:astro-ph/0810.5138 Kaltenegger, L., Traub, W. A., \& Jucks, K. W. 2007, ApJ, 658, 598

Kasdin, N. J., Vanderbei, R. J., Spergel, D. N., \& Littman, M. G. 2003, ApJ, 582, 1147

Kuchner, Crepp, \& Ge 2005, ApJ, 628, 466

Kuchner, M. J., \& Traub, W. A. 2002, ApJ, 570, 900

Lay, O. P., Green, J. J., Hoppe, D. J., \& Shaklan, S. B. 2005, Proc. SPIE, 5905, 148

Lo, A., Noecker, C., Cash, W., \& NWO Study Team 2009, BAAS, 41, 195

Lopez, B., Schneider, J., \& Danchi, W. C. 2005, ApJ, 627, 974

Macintosh, B., et al. 2006, Proc. SPIE, 6272, 62720L

Marois, C., Macintosh, B., Barman, T., Zuckerman, B., Song, I., Patience, J., Lafrenière, D., \& Doyon, R. 2008, Science, 322, 1348

Mawet, D., Riaud, P., Hanot, C., Vandormael, D., Loicq, J., Baudrand, J., Surdej, J., \& Habraken, S. 2007, Proc. SPIE, 6693, 52

Mazumdar, A., et al. 2009, arXiv:0906.3386

Moody, D. C., Gordon, B. L., \& Trauger, J. T. 2008, Proc. SPIE, 7010, 107

Nordgren, T. E., Sudol, J. J., \& Mozurkewich, D. 2001, AJ, 122 2707

Quintana, E. V., Adams, F. C., Lissauer, J. J., \& Chambers, J. E. 2007, ApJ, 660 807

Seager, S., Kuchner, M., Hier-Majumder, C. A., \& Militzer, B. 2007, ApJ, 669, 1279

Shaklan, S. B., \& Green, J. J. 2005, ApJ, 628, 474 
Shaklan, S. B., \& Green, J. J. 2006, Appl. Opt., 45, 5143

Shao, M. 2009, BAAS, 41, 506

Sivaramakrishnan, A., \& Lloyd, J. P. 2005, ApJ, 633, 528

Soummer, R. 2005, ApJ, 618, L161

Spergel, D. N., et al. 2009, in AAS Meeting Abstracts, 213, 458.04

Stapelfeldt, K. R., Krist, J. E., Khan, O., \& ATLAST Concept Study Team 2009, BAAS, 41,343

Thébault, P., Marzari, F., \& Scholl, H. 2008, MNRAS, 388, 1528

Thébault, P., Marzari, F., \& Scholl, H. 2009, MNRAS, 393, L21
Traub, W., Shaklan, S., \& Lawson, P. 2007, in Proc. In the Spirit of Bernard Lyot: The Direct Detection of Planets and Circumstellar Disks in the 21st Century, ed. P. Kalas

Trauger, J. T., \& Traub, W. A. 2007, Nature, 446, 771

Trauger, J. T., et al. 2004, Proc. SPIE, 5487, 1330

Trauger, J. T., et al. 2009, in AAS Meeting Abstracts, 213, 493.01

Wade, R. A., \& Rucinski, S. M. 1985, A\&AS, 60, 471

Wertheimer, J. G., \& Laughlin, G. 2006, AJ, 132, 1995

Wiegert, P. A., \& Holman, M. J. 1997, AJ, 113, 1445 